\title{
WEB-HIPRE - A JAVA APPLET FOR AHP AND VALUE TREE ANALYSIS
}

\author{
Jyri Mustajoki and Raimo P. Hämäläinen \\ Systems Analysis Laboratory \\ Helsinki University of Technology \\ PL 1100, FIN-02150 Espoo, Finland \\ jyri.mustajoki@hut.fi / raimo@hut.fi
}

\begin{abstract}
Web-HIPRE is a Java applet for multiple criteria decision analysis based on the wellknown decision support software HIPRE 3+. Being located on the Internet, Web-HIPRE can be accessed from everywhere in the world. This opens up a completely new era and dimension in decision support. Web-HIPRE provides a common platform for individual and group decision support. The individual models can be processed at the same or at different times and the results can be easily shared and combined. In addition to global access, an essential WWW feature is the possibility to define links to other WWW addresses. These links can refer to any other kind of information such as graphics, sound or video describing the criteria or alternatives. This can improve the quality of decision support dramatically. Web-HIPRE supports several weighting methods including AHP, SMART, SWING, SMARTER and value functions. The results are shown by bar graphs and the sensitivity analysis. Web-HIPRE also supports the use of HIPRE 3+ models. The on-line use of Web-HIPRE will be demonstrated by illustrative examples. WebHIPRE is located on http://www.hipre.hut.fi/ and a slide presentation of the software can be found on http://www.sal.hut.fi/Publications/ppt-files/Web-HIPRE/
\end{abstract}

\section{Introduction}

Web-HIPRE (HIerarchical PREference analysis on the World Wide Web) is an Internet software for multicriteria decision analysis based on the well-known decision support software HIPRE 3+ (Hämalläinen and Lauri, 1995). Web-HIPRE provides implementations of the Analytic Hierarchy Process (AHP) (Saaty, 1980, 1994; Salo and Hämäläinen 1997) and multiattribute value theory (MAVT) (Keeney and Raiffa, 1976) to support the different phases of decision analysis, i.e. structuring of the problem (see e.g. French et al., 1998), prioritization and analyzing the results. Individual models can be integrated into a group model via the Internet. In general, the areas related to Web-HIPRE include decision making, group collaboration, and computer support (see Figure 1). Web-HIPRE is the first globally available decision analytical software on the Internet.

In addition to global access, the Internet offers information sources, which can be very useful in the decision support process. In Web-HIPRE this information can be utilized by linking the elements of the hierarchy with the WWW pages. They can contain any kind of multimedia material such as videos, pictures, sound, virtual tours and so on, which can help the decision maker to more accurately define his/her attributes and preferences. Figure 2 presents the WWW page for the example problem of selecting a cellular phone. The same example is used throughout the presentation to demonstrate different features of Web-HIPRE. All three cellular phones have their own WWW pages, which are linked with the alternative elements of the hierarchy.

In the Systems Analysis Laboratory, there is also a variety of other decision support systems on the

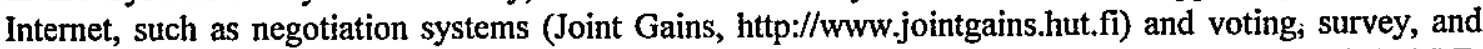
group collaboration systems (Opinions-Online, http://www.opinion.hut.fi). Experiences on Web-HIPRE, and also on these other projects, have showed that the Internet can open up considerable possibilities in different areas, such as decision analysis, and especially in group collaboration. 



Figure 1. Areas related to Web-HIPRE

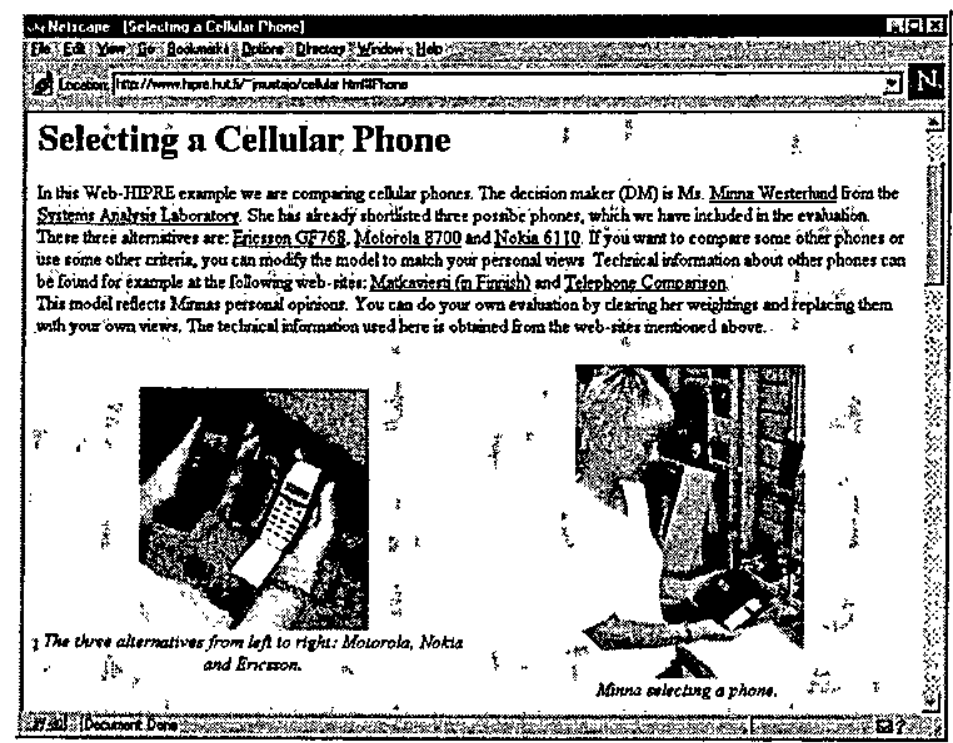

Figure 2. WWW page explaining the cellular phone example

Web-HIPRE can be accessed from any location with a Java-enabled WWW browser (e.g. Netscape or Internet Explorer) connected to the Internet. When using Web-HIPRE, the browser loads the applet in its local memory, from where it is operated. When the browser is closed, no files remain on user's computer. The software can also be installed in an independent computer and run locally. Web-HIPRE supports the use of HIPRE $3+$ models.

\section{Prioritization Methods}

The methods used in Web-HIPRE are based on the analytic hierarchy process (AHP) and on multiattribute value theory (MAVT). The original form and some variants of AHP are supported. In the area of MAVT, Web-HIPRE supports direct weighting, SMART (Edwards, 1977; von Winterfeldt and Edwards, 1986; Edwards and Barron, 1994), SWING (von Winterfeldt and Edwards, 1986) and the rank based SMARTER technique (Edwards and Barron, 1994). On the lowest level, value scores can be given directly or by using value furictions. 
The problem is structured visually with the graphical user interface (GUI). Figure 3 presents the hierarchy of the example model for selecting a cellular phone.

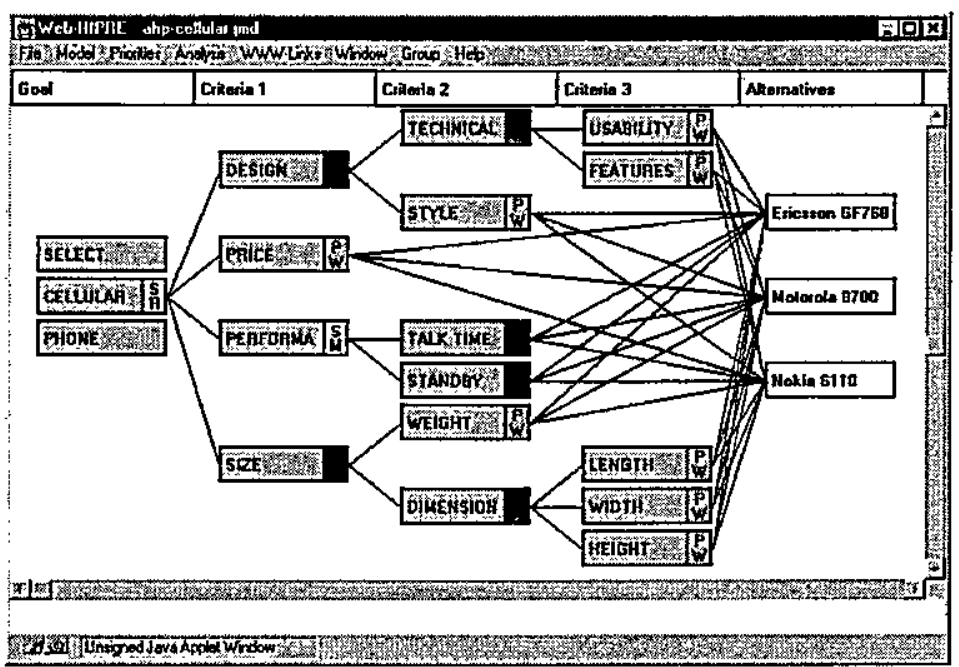

Figure 3. Hierarchy window. Different weighting methods can be used and the selected methods are shown by abbreviations

\section{The Analytic Hierarchy Process (AHP)}

Besides the original form of AHP, Web-HIPRE also supports continuous preference scale. Preferences can be given, for example, graphically with the slider or numerically by typing a value (Figure 4). When all the pairwise comparisons have been given, the elicited weights for each attribute are shown by numbers and bar graphs.

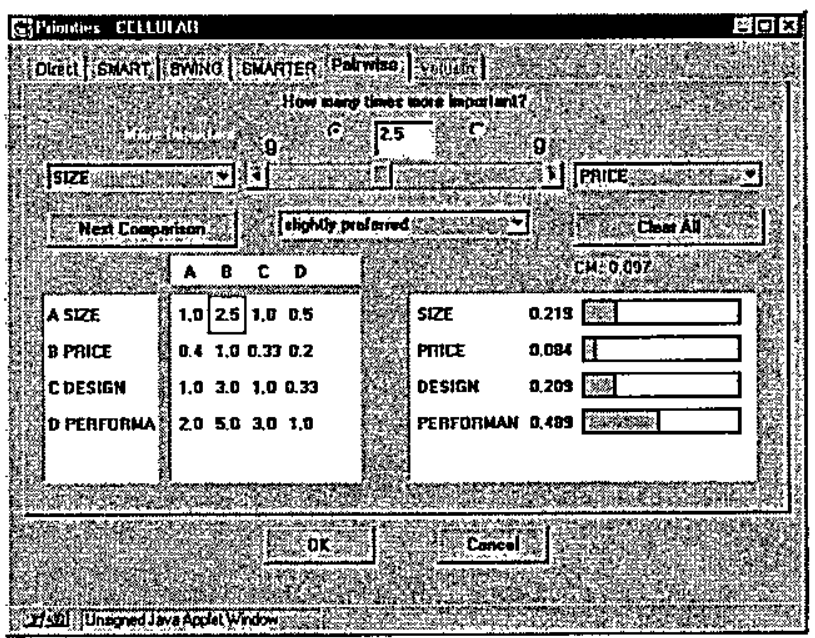

Figure 4. AHP weighting window

Verbal statements can also be used to define preferences by choosing an appropriate expression from the list. Web-HIPRE uses the original mapping from words to ratios, which is: equal preference $=1$, moderate preference $=3$, strong preference $=5$, very strong preference $=7$, and extreme preference $=9$ (Saaty, 1980). However, it has been shown that, for example, the use of the 9/9-9/1 scale (Ma and Zheng, 1991) or the balanced scale (Salo and Hämalläinen, 1997) gives a better equivalency to the verbal expressions 
(Pöyhönen et al., 1997). These variant scales can be used by selecting the corresponding ratios from the continuous scale.

However, the original consistency ratio (CR) of AHP (Saaty, 1980) is not applicable with the general scales. When using different discretization of the scale or a continuous scale, a scale-invariant consistency measure should be used. In Web-HIPRE the consistency measure (CM) (Salo and Hämälainen, 1997) is used.

In the consistency measure (CM), inconsistent replies are transformed into a non-empty set of feasible preferences and the properties of this set can be used to measure the inconsistency of the original matrix. More precisely, the consistency measure is

$$
C M=\frac{2}{n(n-1)} \sum_{i>j} \frac{\bar{r}(i, j)-\underline{r}(i, j)}{(1+\bar{r}(i, j))(1+\underline{r}(i, j))}
$$

where $\bar{r}(i, j)=\max _{\mathbf{k}} a(i, k) a(k, j)$ stands for the extended bound of the comparison matrix element $a(i, j)$ (the element in the $i$ th row and the $j$ th column) (Salo, 1993), and $r(i, j)$ is the inverse of $\bar{r}(j, i)$. Thus, the consistency measure is an indicator of the size of this extended region formed by the set of local preferences such that $w_{\mathrm{j}} \leq \bar{r}(i, j) w_{\mathrm{j}}$ for all $i, j \in\{1, \ldots, n\}$. This measure increases with the inconsistency of the elements of the pairwise comparisons matrix.

\section{Multiattribute Value Theory (MAVT)}

In MAVT the problem is structured into a hierarchical form similarly as in AHP. The objective is to obtain overall value scores for each alternative. The value scores are composed of the ratings of the alternatives with respect to each attribute and of the weights of the attributes.

By using an additive value function, the overall value score of an alternative $x$ is

$$
v(x)=\sum_{i=1}^{n} w_{i} v_{i}\left(x_{i}\right),
$$

where $v_{i}\left(x_{i}\right)$ is the component value of an attribute rating $x_{i}$ and $w_{i}$ is the weight associated with an attribute $i$. The component value functions $v_{i}(\odot)$ and the weights $w_{i}$ get values between 0 and 1 , and the weights are normalized to sum up to one. The weight $w_{i}$ indicates the relative importance of the event of an attribute $i$ changing from its worst level to its best level compared to the corresponding changes on the other attributes.

In MAVT the weights of the attributes can be given directly, or some sophisticated method, for example, SMART, SWING or SMARTER, can be used. On the lowest level, value scores can be given directly or, for example, value functions can be used to transform the ratings of the alternatives into the value scores.

\section{Combined Use of the Methods}

A unique feature of Web-HIPRE is the possibility to use different weighting methods in one hierarchy. Thus, under each element of the hierarchy the decision maker can select the most suitable method. He/she can apply different methods, but only one of them is active. All the prioritizations made by the other methods are stored, which makes the comparison of different weighting methods easy.

AHP and MAVT methods can be combined freely as such in criteria weighting. On the alternative level, AHP weights can be converted to the compatible $0-1$ value scale by setting the lowest priority weight is to zero, the highest priority weight to one, and scaling the intermediate weights proportionally to this scale (Dyer, 1990). More precisely, the converted weight $w_{\mathrm{ci}}$ for the alternative $i$ is

$$
w_{c t}=\frac{w_{l}-w_{\min }}{w_{\max }-w_{\min }}
$$


where $w_{\max }=\max \left(w_{1}, \ldots, w_{\mathrm{n}}\right)$ and $w_{\min }=\min \left(w_{1}, \ldots, w_{\mathrm{n}}\right)$ are the original maximum and minimum AHP weights. These converted AHP weights can now be treated as value scores. Alternatively, value scores can be normalized to sum up to one to make them compatible with the AHP weights.

\section{Analyzing the results}

In Web-HIPRE the composite priorities of the alternatives are shown by bar graphs (Figure 1) or by numerical values. Bars can be divided to segments indicating the importance of criteria. The composite priorities can also be calculated with any element acting as a goal. Sensitivity of the criteria can be analyzed in a separate window.
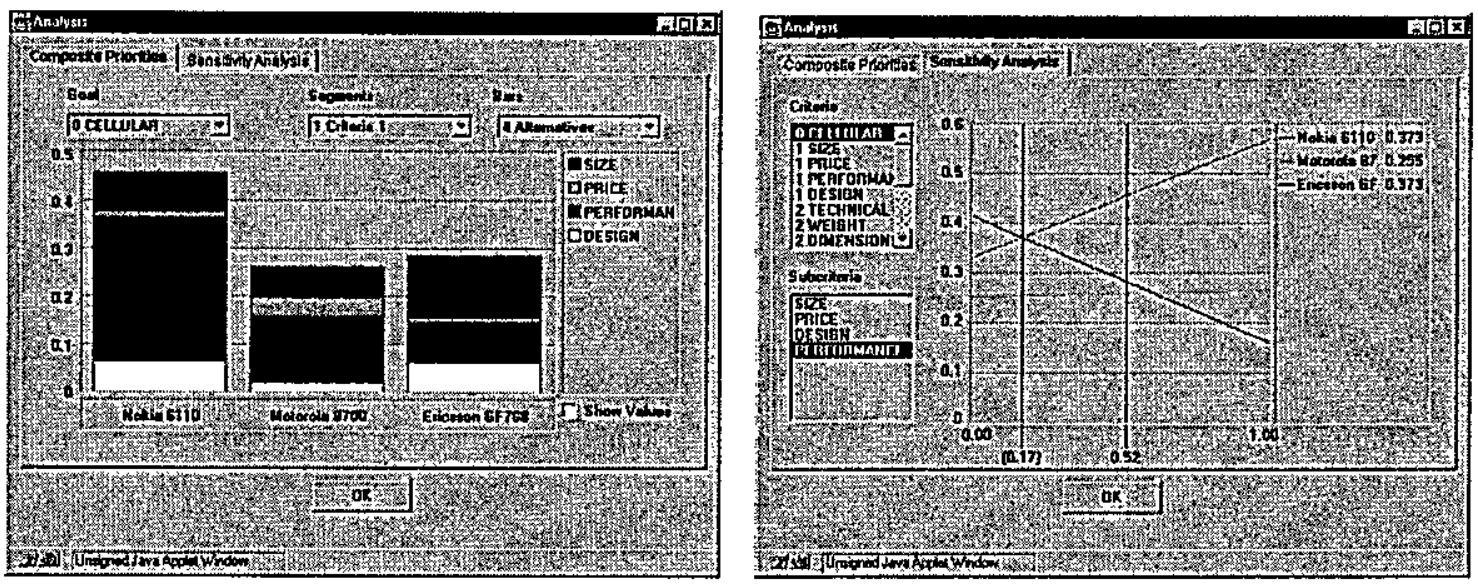

Figure 1. Composite priorities (left) and sensitivity analysis windows

\section{Group Decision Making}

There are different ways of combining individual priorities into group priorities. In preference programming (Salo and Hämäläinen 1995), it is possible to define intervals for the weight ratios instead of exact number estimates. Originally, the intervals were applied to denote uncertainty of an individual decision maker, but they can also be adapted into group decision making by forming an interval which includes the weight ratios of all the group members (see e.g. Hämalalinen and Pöyhönen, 1996). As the local weights are presented as intervals, the overall priorities are also intervals. Preference programming is supported, for example, by the Winpre software (Hämäläinen and Helenius, 1997).

Web-HIPRE can be used to support global distributed group decision making by creating a group model, where each decision maker is graphically represented by an element in the group hierarchy. Such an element actually presents the whole hierarchy of an individual decision maker. The composite group priorities are generated as a weighted sum of the individual priorities for the alternatives (Dyer and Forman, 1992; Keeney and Raiffa, 1976), which are obtained from the individual models via the Internet.

Another way to support group decision making in Web-HIPRE is to use it simply as a collaboration platform. Web-HIPRE models can be made available to other decision makers, and by analyzing the models of other decision makers a better understanding of their objectives can be achieved.

\section{References}

Dyer, J.S., 'Remarks on the Analytic Hierarchy Process' (and replies by P.T. Harker, T. Saaty, and L.G. Vargas), Management Science, 36, 3, 1990, pp. 249-275. 
Dyer, R.F. and Forman, E.H., 'Group decision support with the Analytic Hierarchy Process', Decision Support Systems, 8, 1992, pp. 99-124.

Edwards, W., 'How to Use Multiattribute Utility Measurement for Social Decisionmaking', IEEE Transactions on Systems, Man and Cybernetics, 7, 5, 1977, pp. 326-340.

Edwards, W. and Barron, F.H., 'SMARTS and SMARTER: Improved Simple Methods for Multiattribute Utility Measurement', Organizational Behavior and Human Decision Processes, 60, 1994, pp. 306-325.

French, S., Simpson, L., Atherton, E., Belton, V., Dawes, R., Edwards, W., Hämäläinen, R.P., Larichev, O., Lootsma, F., Pearman, A. and Vlek, C., 'Problem Formulation for Multi-Criteria Decision Analysis: Report of a Workshop', Journal of Multi-Criteria Decision Analysis, 7, 1998, pp. 242-262.

Hämălăinen, R.P. and Helenius, J., 'Winpre - Workbench for Interactive Preference Programming', Systems Analysis Laboratory, Helsinki University of Technology, 1997. http://www.sal.hut.fi/ Downloadables/winpre.html (Slide presentation at http:/www.sal.hut.fi/Publications/ppt-files/WINPRE/)

Hämäläinen, R.P. and Lauri, H., 'HIPRE 3+ User's Guide', Systems Analysis Laboratory, Helsinki University of Technology, 1995. Downloadable at http://www.sal.hut.fi/Downloadables/hpdemo.html

Hämäläinen, R.P. and Pöyhönen, M., 'On-Line Group Decision Support by Preference Programming in Traffic Planning', Group Decision and Negotiation, 5, 1996, pp. 485-500.

Keeney, R.L. and Raiffa, H., 'Decisions with Multiple Objectives: Preferences and Value Tradeoffs', John Wiley \& Sons, Inc., 1976.

Ma, D. and Zheng, X., '9/9-9/1 scale method of AHP', Proc. 2nd Int. Symp. on the AHP, Pittsburgh, PA, Vol. I, 1991, pp. 197-202.

Nunamaker, J.F., 'Future research in group support systems: needs, some questions and possible directions', Int. J. Human-Computer Studies, 47, 1997, pp. 357-385.

Poyhönen, M., Hämalainen, R.P. and Salo, A.A., 'An Experiment on the Numerical Modelling of Verbal Ratio Statements', Journal of Multi-Criteria Decision Analysis, 6, 1997, pp. 1-10.

Saaty, T.L., 'The Analytic Hierarchy Process', McGraw-Hill, Inc., 1980.

Saaty, T.L., 'Highlights and critical points in the theory and application of the Analytic Hierarchy Process', European Journal of Operational Research, 74, 1994, pp. 426-447.

Salo, A.A., 'Inconsistency analysis by approximately specified priorities', Math. Comput. Modelling, 17, 1993, pp. 123-133.

Salo, A.A. and Hämăläinen, R.P., 'Preference programming through approximate ratio comparisons', European Journal of Operational Research, 82, 1995, pp. 458-475.

Salo, A.A. and Hämalainen, R.P., 'On the Measurement of Preferences in the Analytic Hierarchy Process' (and comments by V. Belton, E. Choo, T. Donegan, T. Gear, T. Saaty, B. Schoner, A. Stam, M. Weber, B. Wedley), Journal of Multi-Criteria Decision Analysis, 6, 1997, pp. 309-343.

von Winterfeldt, D. and Edwards, W., 'Decision Analysis and Behavioral Research', Cambridge University Press, 1986. 